\section{$8^{\text {th }}$ International Conference on Civil and Architecture Engineering \\ ICCAE-8-2010}

\title{
A Framework for the Integration of Remote Sensing Systems for 3D Urban Mapping
}

By
Ayman Habib*
Changjae Kim*
Eunju Kwak ${ }^{*}$

\section{$\underline{\text { Abstract: }}$}

As one of the fields of civil and architecture engineering, 3D urban mapping plays a vital role in various applications such as urban planning, surveillance, virtual reality, virtual tourism, and military training. In this regard, the interest in $3 \mathrm{D}$ urban mapping technologies is rapidly increasing within the surveying and photogrammetric community. Such an interest is also motivated by the advent of new technologies, which enable accurate and practical 3D urban mapping. In other words, the proliferation of direct geo-referencing, digital imaging system (including medium-format digital camera) and LiDAR (Light Detection And Ranging) provide the respective research body with the potential to satisfy the detail level and complexity needed by the above applications. Hence, there must be a framework for integrating these different kinds of sensors. The proposed framework in this paper consists of three main components: 1) Quality Assurance/Quality Control; 2) Co-registration; and 3) Element Matching. More specifically, quality assurance of the mapping process and quality control of delivered data/products are the first components of the proposed framework. Quality assurance encompasses management activities to ensure that a process, item, or service is of the quality needed by the user. The key activity in the quality assurance is the system calibration procedure. After the calibration of the involved systems, quality control procedures determine whether the desired quality has been achieved through internal and external evaluation. As the second component of the framework, a registration procedure is conducted to ensure that the datasets from different systems are georeferenced with respect to a common reference frame. After the registration procedure is

\footnotetext{
* Department of Geomatics Engineering, University of Calgary, Calgary, Canada
} 
completed, matching between different information from different systems is carried out to derive realistic 3D urban mapping that takes advantage of the synergistic characteristics of the available datasets. For example, the spectral information from a digital imaging system can be related to the positional information from LiDAR. The paper will illustrate the main components and the necessary activities of the proposed framework with the help of a real dataset.

\section{Keywords:}

3D urban mapping, Quality Assurance/Quality Control, co-registration and element Matching

\section{Introduction:}

The United Nations predicts that the number of dwellers in urban areas will rise to almost 5 billion inhabitants by 2030 in the report entitled 'State of the World Cities $2006 / 7$ '. Such a population will constitute roughly $62 \%$ of the global population at that time. To successfully control social and environmental situations that might arise from this rapid urbanization, decision makers in various fields must have access to accurate and up-to-date geo-spatial information in a timely manner and at a reasonable cost. It has been, hence, recognized that the research and academic communities should develop practical and accurate 3D urban mapping tools for assisting these decision makers in exploiting the influx of geo-spatial datasets from multi-sensory systems. Hence, there is a significant need for the integration of the different sensory systems. In this regard, this research proposes an integration framework for urban mapping consisting of three main components: 1) Quality Assurance/Quality Control; 2) Co-registration; and 3) Element Matching. Such components will be addressed sequentially in the following sections both with theoretical explanations and with the intermediate results using real datasets. The last section will summarize the gained knowledge from this research. One should note that the intermediate results in each section are acquired by photogrammetric and LiDAR data capturing systems over the University of Calgary campus in Calgary, Alberta, Canada. More specifically, the LiDAR dataset was captured by an Optech 3100 system (from two different mapping missions at two different flying heights, $1000 \mathrm{~m}$ and $1400 \mathrm{~m}$ ) and the photos (with a scale of 1:5000) were captured by an analog camera RC30.

\section{Quality Assurance and Control of Photogrammetric and LiDAR Data:}

Quality assurance and control of photogrammetric and LiDAR data are considered to be the first necessary component of the proposed framework for 3D urban mapping. 
Quality assurance encompasses management activities to ensure that a process, item, or service is of the quality needed by the user. It deals with creating management controls that cover mission planning, implementation, and review of data collection activities. The key activity in the quality assurance is the system calibration procedure. Internal camera characteristics, which are usually known as Interior Orientation Parameters (IOP), are derived through the process of camera calibration. A typical LiDAR system, on the other hand, consists of three main components: a Global Positioning System (GPS) system to provide position information, an Inertial Navigation System (INS) unit for attitude determination, and a LASER system to provide range (distance) information between each LASER firing point and corresponding ground point. The quality of the surface derived using a LiDAR system depends on the accuracy of the data derived from the three components mentioned above and the calibration parameters relating these components (i.e., mounting parameters). The proposed framework adopted the camera and LiDAR system calibration methodologies recommended by Habib et al. [1], [2].

After the calibration of both systems, quality control procedures determine whether the desired quality has been achieved or not. Photogrammetric quality control is investigated through the precision of the reconstructed object points and check point analysis. Internal and external LiDAR quality control can be implemented by evaluating the degree of consistency among the LiDAR footprints in overlapping strips and by utilizing LiDAR control targets, respectively. Four different quality control methods for LiDAR quality control analysis were suggested for this framework [3]. The first two methods utilize derived conjugate linear features and patches from overlapping strips, respectively. The basic concept of these methods is that conjugate linear features and patches from different strips should be collinear and coplanar in the absence of biases, respectively. The third method called the ICPatch method uses a TIN generated from a strip and original LiDAR points from the other strip while assuming a point-to-patch correspondence. The last method called the ICPoint method assumes a point-to-point correspondence between original LiDAR points from two different strips. Once the biases between different strips have been detected through the quality control methods, the LiDAR strips can be adjusted using a 3D similarity transformation. The following figure shows an example of the adjustment of LiDAR strips based on the transformation parameters estimated using the quality control methods. 

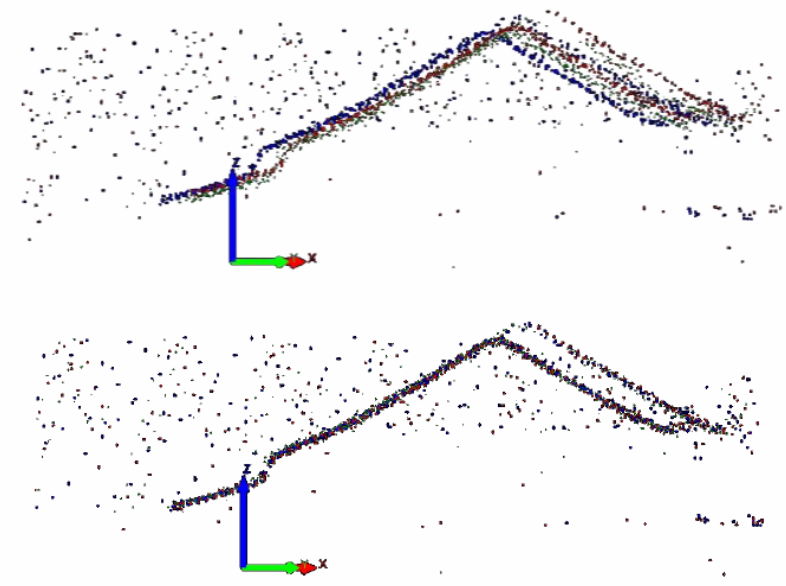

Figure (1): LiDAR point clouds for two buildings before (top) and after (bottom) the LiDAR quality control procedures, note that each color indicates a different strip

Once the quality assurance and quality control of photogrammetric and LiDAR data have been conducted, photogrammetric and LiDAR data can be used with confidence to move on to the next component. Since this research deals with two different data types, they must be registered to a common reference frame. Hence, next section explains the co-registration techniques to be used, which incorporate both linear and areal features.

\section{Co-reqistration:}

Only after ensuring that the photogrammetric and LiDAR datasets are geo-referenced with respect to a common reference frame, can the synergic properties of the two datasets be fully utilized [4]. Traditionally, photogrammetric geo-referencing is either done indirectly using Ground Control Points (GCP), or directly using GPS/INS units onboard the imaging platform [5]. LiDAR geo-referencing, on the other hand, is done directly using the GPS and INS components of the LiDAR system. When GCP-based or GPS/INS-based photogrammetric geo-referencing is conducted, there might be biases between the two reference frames for photogrammetric and LiDAR data. The biases can be caused by utilizing different GPS base stations for photogrammetric and LiDAR data acquisition. In addition, utilization of GCP for photogrammetric geo-referencing is expensive. In this framework, the LiDAR data is used as the source of control for photogrammetric geo-referencing; thus assuring proper co-registration.

Since LiDAR footprints are irregularly distributed, it is almost impossible to assume point-to-point correspondence between photogrammetric and LiDAR data. Georeferencing techniques that use existing point-based bundle adjustment procedures and incorporate linear and areal features were introduced by Habib et al. [6]. The key point is that these techniques simply manipulate the weight matrices associated with the 
points belonging to these features without modifying the existing point-based bundle adjustment procedures. More detailed explanations follow in Sections 3.1 and 3.2.

\subsection{Point-based incorporation of linear features:}

Here, geo-referencing is carried out by incorporating linear features in photogrammetric triangulation. Image and object space lines are each defined by two end points, extracted from the image and object space (i.e., LiDAR), respectively. There is no need for correspondence between the image and object space points along the linear features, as shown in Figure 2. This is because the weight matrix of each image point involved is restricted in this approach. Only useful information along the normal direction to the image space line is utilized. In other words, the point is free to move along the image space line. A new weight matrix, which controls the movement of the point, can be applied in the point-based solution using the collinearity equations. The minimum requirement for control linear features to establish the geo-referencing parameters for the involved imagery is to have two non-coplanar lines [7].

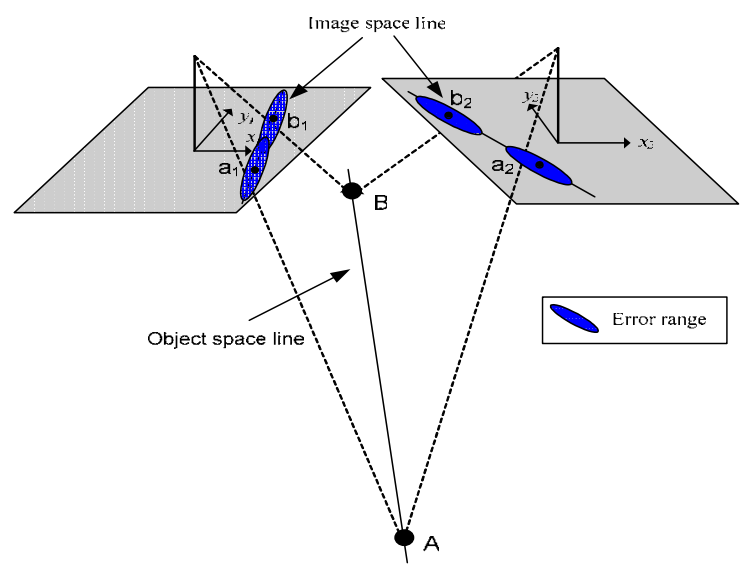

Figure (2): Representation of image and object space lines for the point-based incorporation of linear features

\subsection{Point-based incorporation of areal features:}

As another alternative for geo-referencing, an approach that incorporates areal features extracted from LiDAR data is introduced here. The vertices of conjugate patches should be observed in at least two overlapping images. The number of points selected in the corresponding LiDAR patch should be equivalent to the number of vertices defined in the imagery. One should note that there is no need for correspondence between image and object space points, as shown in Figure 3. The weight matrix of each patch vertex in the object space is restricted in this approach. Only useful information along the normal 
direction to the object plane is utilized. In other words, the point is free to move along the object plane. A new weight matrix, which controls the movement of the point, can be applied in the point-based solution using the collinearity equations. The minimum requirement for areal control features to establish the geo-referencing parameters for the involved imagery is to have four planar patches (three patches parallel to the planes XY, $Y Z$, and ZX, respectively and a parallel patch to one of the planes XY, YZ, or ZX). However, the probability of having vertical patches in airborne LiDAR data is not high. Therefore, instead of working with vertical patches, one can use tilted patches with different slopes and aspects [7].

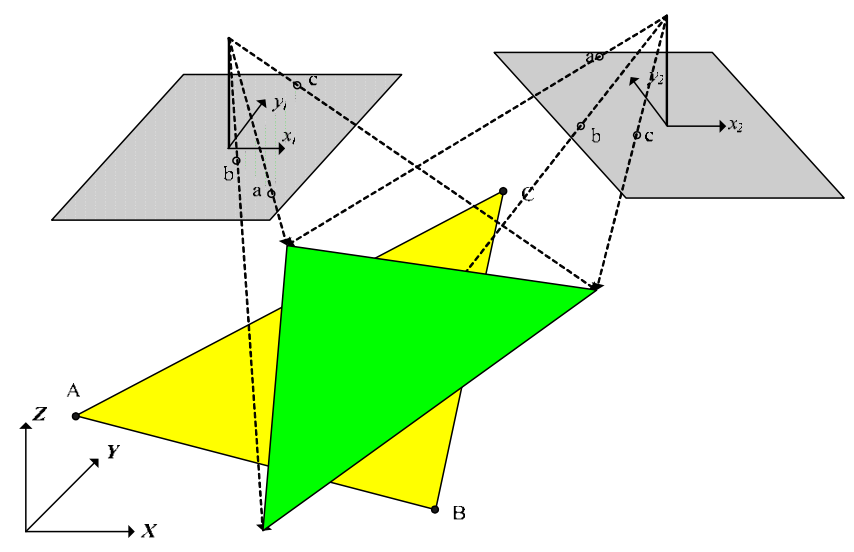

Figure (3): Representation of corresponding patches in the image and object space

To evaluate the performances of the co-registration methods with different sources of control information, three true orthophotos are produced by using a perspective image (Figure 4(a)), a digital surface model, and three sets of EOPs resulting from using GCPs, patches derived from LiDAR, and lines derived from LiDAR as sources of control. By examining these true orthophotos, it is clear that two of the generated true orthophotos using LiDAR patches and LiDAR lines are compatible (Figure 4(c) and (d)). Moreover, the true orthophotos generated using LiDAR patches or lines appear to be more accurate than the one generated using GCPs. This can be observed in the orthophotos, where there are more traces of building boundaries in the latter true orthophoto (Figure 4(b)). For this reason, the features derived from LiDAR data can be utilized as a source of control for photogrammetric geo-referencing to assure better coregistration of the photogrammetric and LiDAR data. 


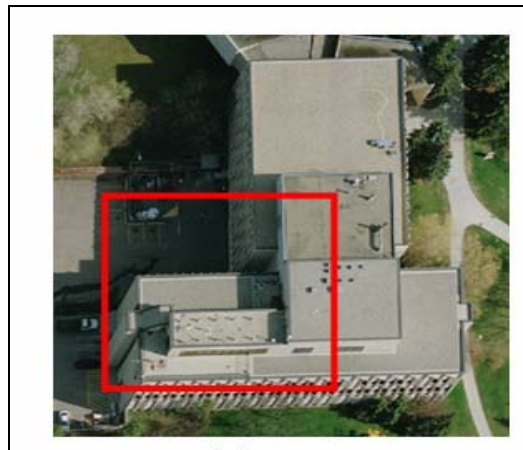

(a)

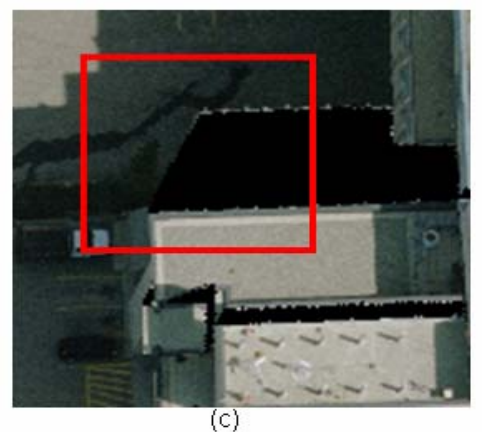

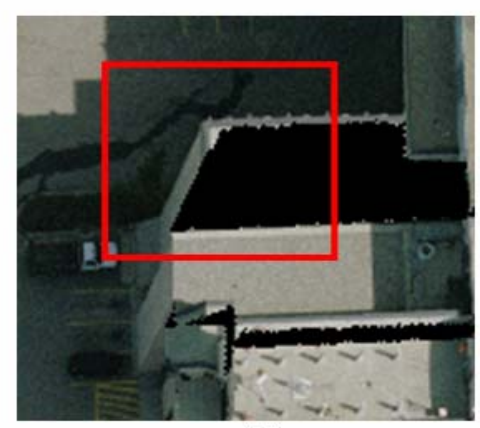

(b)

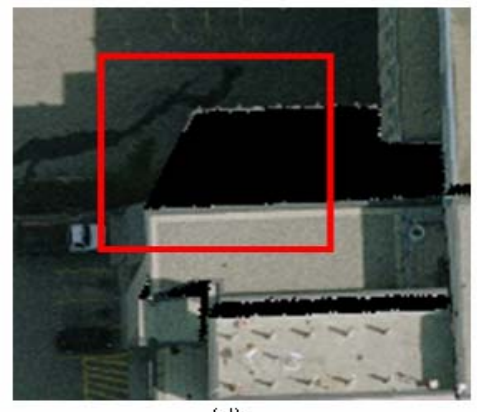

(d)

Figure (4): Perspective image (a), and orthoimage using the geo-referencing parameters from GCPs (b), LiDAR patches (c), and LiDAR lines (d), as the source of control

\section{Spectral and Positional Element Matching:}

Once the photogrammetric dataset has been aligned to the reference frame of the LiDAR data, the image spectral (i.e., color values) and LiDAR positional (i.e., X, Y, Z) information from the two datasets are ready to be linked to one another in the element matching process. One should note that orthophoto production aims at relating the image spectral and LiDAR positional information correctly through the elimination of sensor tilt and terrain relief effects from the captured perspective imagery. Differential rectification has been widely used for orthophoto generation [8], [9]. When dealing with large scale imagery over urban areas, however, differential rectification produces significant artifacts in the form of double mapped areas at the vicinity of abrupt surface changes [10]. Therefore, true orthophoto generation methodologies focus on the elimination of the double mapped areas. The basic principle of these methodologies is the identification of occluded areas, which are caused by relief displacements associated with vertical structures in the object space.

True orthophoto generation is mainly concerned with visibility analysis, which has been studied in computer graphics, computer vision, photogrammetry, remote sensing, and 
telecommunications. To overcome false visibility and false occlusion problems associated with the majority of existing true orthophoto generation techniques, which is based on Z-buffer algorithm [11] - [16], the angle-based true orthophoto generation method is introduced in this research.

In the angle-based true orthophoto generation method, the presence of occlusions can be discerned by sequentially checking the off-nadir angles to the lines of sight connecting the perspective center to the Digital Surface Models (DSM) points along a radial direction starting from the object space nadir point. In the remainder of this section, the off-nadir angle to the line of sight will be denoted as the $\alpha$ angle (Figure 5).

Since there is no relief displacement associated with the object space nadir point, one can assure that this point will be always visible in the acquired image. As one moves away from the object space nadir point, it is expected that the $\alpha$ angle will increase gradually. As long as there is an increase in the $\alpha$ angle as one moves away from the nadir point, the DSM cells along the radial direction will be visible in the image in question. On the other hand, occlusions will take place whenever there is an apparent decrease in the $\alpha$ angle while proceeding away from the nadir point. This occlusion will persist until the $\alpha$ angle exceeds the angle associated with the last visible point. Figure 5 illustrates the mechanics of using the off-nadir angle to the line of sight in detecting occluded areas by considering a vertical profile through the perspective center. As can be seen in this figure, moving away from the nadir point will be accompanied by an increase in the $\alpha$ angle until one reaches the object point $A$, thus indicating that there is no occlusion up to this point. However, when considering the object point $\mathrm{B}$, one would notice that $\alpha \mathrm{B}$ is smaller than $\alpha \mathrm{A}$, which indicates that point $\mathrm{B}$ is occluded by $\mathrm{A}$. For the object point $\mathrm{C}$, it is obvious that $\alpha \mathrm{C}$ is greater than $\alpha \mathrm{A}$, which indicates that $\mathrm{C}$ is visible. 


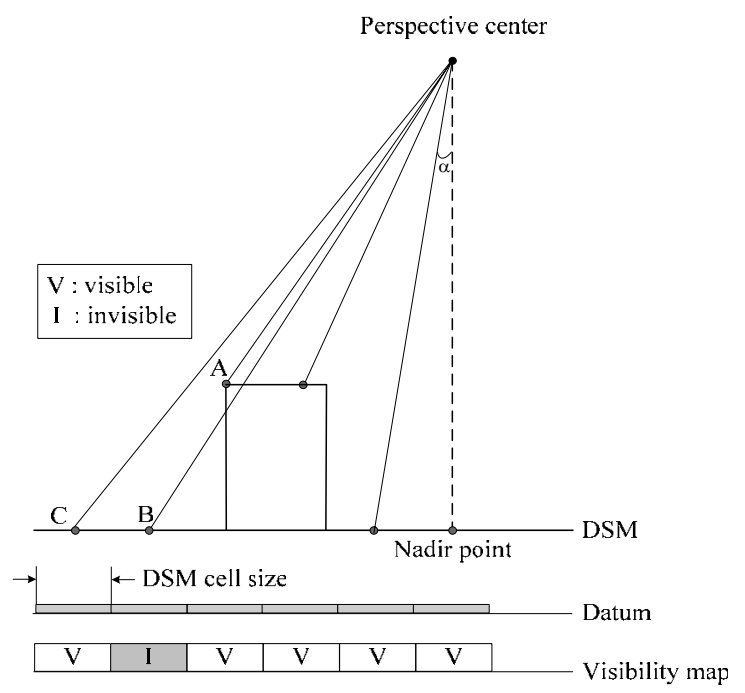

Figure (5): Using the off-nadir angle to the line of sight as a means of detecting occlusions

In contrast to other true orthophoto generation techniques, the performance of the anglebased true orthophoto generation method does not depend on the relative relationship between the DSM cell size and the GSD of the imaging sensor because such a method of occlusion detection does not incorporate the internal characteristics and attitude of the imaging sensor [13] - [15].

To compare the true orthophotos resulting from the angle-based method and Z-buffer method more carefully, one can refer to Figures 6(a) and (b), respectively. The true orthophoto produced using the angle-based method (Figure 6(a)) does not introduce any false visibility or occlusions. On the other hand, the true orthophoto produced using Zbuffer method still has false visibility in the double mapped areas. Figure 6(c) shows the true orthophoto (based on the angle-based method) after filling occluded cells using overlapping images. Smooth transition of the gray values between overlapping true orthophotos can be recognized in Figure 6(c) as a result of reliable quality assurance/quality control and geo-referencing procedures. The true orthophoto generated can be draped on top of the DSM to produce the 3D visualization of the surfaces in Figure 7. 


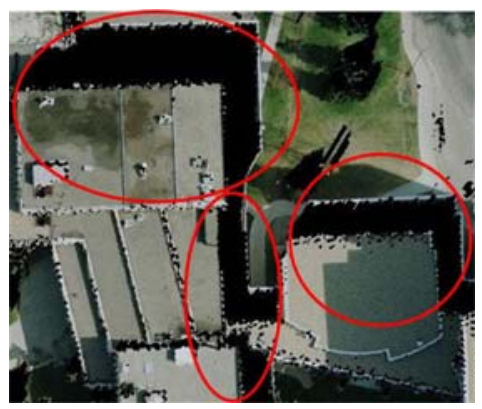

(a)

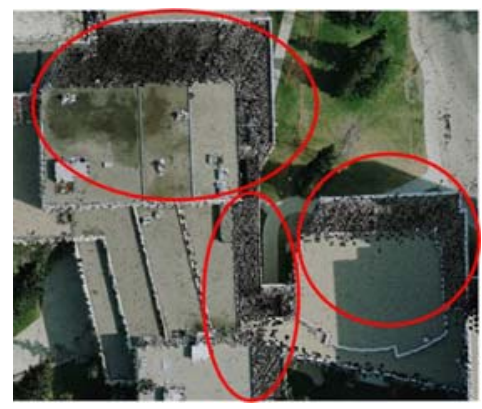

(b)

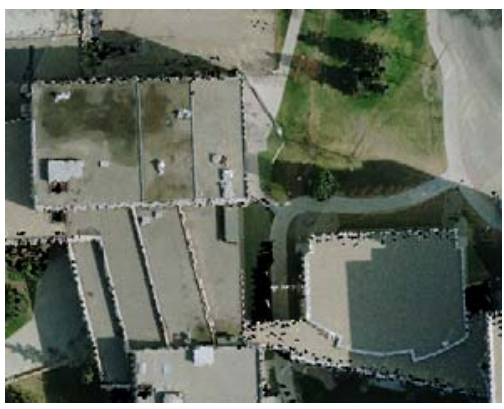

(c)

Figure (6): The areas in the true orthophotos created using angle-based method (a), using Z-buffer method (b), and after filling the occlusions using overlapping true orthophotos (based on the angle-based method) (c)

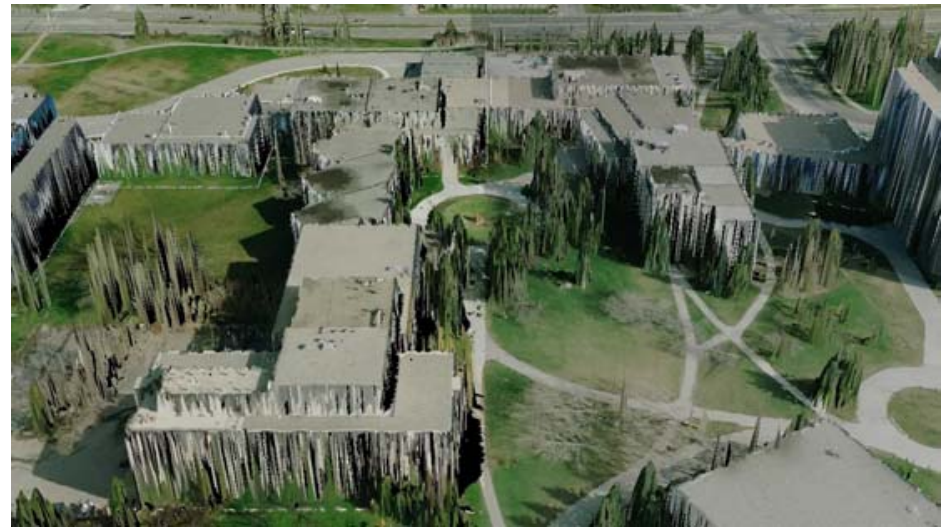

Figure (7): 3D perspective view after draping the generated true orthophoto on top of the DSM

At this stage, one should note that the produced 2D and 3D visualizations, created using the proposed framework, are not yet of sufficient quality, especially, near building boundaries (Figures 6(c) and 7). Such degradation arises due to incomplete surface models which are generated from the LiDAR data with the irregular and sparse nature. Hence, there is a need to enhance the surface models by introducing Digital Building Models (DBM) with precise building boundary information.

One should note that LiDAR data can provide overall trend but not accurate details on the shape of the building. Conversely, imagery contains richer building boundary information, such as edge lines and colour attributes, which provide more details on the shape of the buildings. Hence, building model generation will be based on the 
integration of photogrammetric and LiDAR data. The warped imagery, which is a new concept in the integration of LiDAR data and imagery, is introduced instead of using the original imagery directly. More specifically, warped images are generated through the simple projection of the original images onto the rooftop plane of a given building hypothesis generated from LiDAR data. Using the warped images, precise building boundaries are generated after 3D straight lines are detected, matched, grouped, and filtered. Once the precise DBM has been produced, the enhanced surface model is generated using the original DSM and the precise building boundary information from DBM. Figures 8(a) and (b) show the true orthophoto and 3D visualization generated using a new DSM obtained by adding the DBM to the original DSM, respectively. Compared to Figures 6(c) and 7, the level of quality of the product is significantly improved in Figures 8(a) and (b).

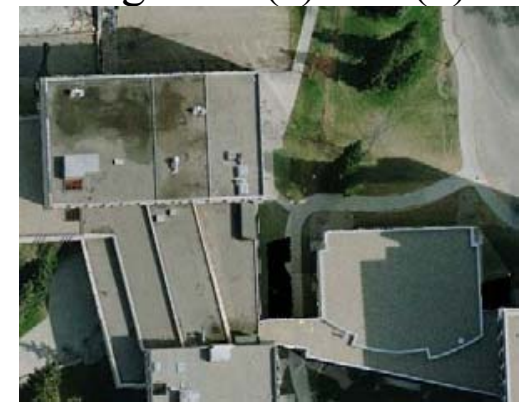

(a)

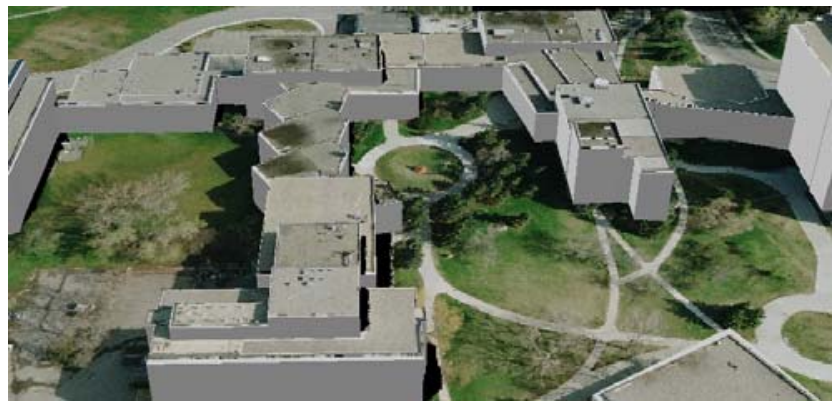

(b)

Figure (8): A true orthophoto (a) and a 3D visualization (b) created using a DSM obtained by adding the DBM to the original DSM

\section{Conclusions and recommendations for future work:}

This research proposed a new framework consisting of three key components for reliable 3D urban mapping products. Quality assurance and quality control procedures ensured that the photogrammetric and LiDAR data can be used with confidence for urban mapping applications. Afterwards, highly reliable co-registration results were achieved by utilizing LiDAR data as a source of control for photogrammetric georeferencing. Once the photogrammetric and LiDAR datasets are geo-referenced with respect to a common reference frame, the spectral and positional elements can be matched though the proposed true orthophoto generation algorithm without false visibility and false occlusions. In summary, the framework proposed in this research made it possible to produce reliable 3D urban mapping products. As a future work, the level of automation will be improved to increase the efficiency of the proposed framework. Also, the missing texture on the walls should be recovered by utilizing oblique or terrestrial images to improve the quality of the 3D visualization. The level of 
detail of the constructed building model though the proposed framework might be recognized as LOD2 according to Open Geospatial Consortium (OGC) standards. Hence, this approach should be improved and extended to reach LOD3, which includes detailed architecture model, through the integration of Terrestrial Laser System (TLS) and close range imaging systems.

\section{Acknowledqements:}

Acknowledgement must be given to the Korean Electronics and Telecommunications Research Institute (ETRI), GEOmatics for Informed Decisions (GEOIDE - IV-17 and SII-72), and National Science \& Engineering Research Council of Canada (NSERC) (Discovery Grant).

\section{References:}

[1] Habib, A., Quackenbush, P., Lay, J., Wong, C., and Al-Durgham, M., 2006. Camera calibration and stability analysis of medium-format digital cameras. Proceedings of SPIE - 6312, Applications of Digital Image Processing XXIX, 11 pages.

[2] Habib, A., Bang, K., Kersting, A., Lee, D., 2009. Error Budget of LiDAR Systems and Quality Control of the Derived Data. Photogrammetric Engineering and Remote Sensing, 75(9): 1093-1108.

[3] Al-Durgham, M. M., 2007. Alternative Methodologies for the Quality Control of LiDAR Systems. Thesis UCGE Reports Number 20259, University of Calgary, AB, Canada.

[4] Habib, A., and T. Schenk, 1999. New approach for matching surfaces from laser scanners and optical sensors, International Archives of Photogrammetry and Remote Sensing, 32: 55-61.

[5] Cramer, M., Stallmann, D., and Haala, N., 2000. Direct Georeferencing Using GPS/Inertial Exterior Orientations for Photogrammetric Applications, International Archives of Photogrammetry and Remote Sensing, 33(B3): 198-205.

[6] Habib, A., Jarvis, A., Kersting, A., Alghamdi, Y., 2008. Comparative Analysis of Geo-referencing Procedures Using Various Sources of Control Data, XXIst ISPRS proceedings, 3-11 Jul, 2008, Beijing, China, 27: 1147 - 1152. 
[7] Ghanma, M., 2006. Integration of Photogrammetry and LIDAR, Ph.D. thesis: University of Calgary, Calgary, Canada.

[8] Konecny, G., 1979. Methods and possibilities for digital differential rectification. Photogrammetric Engineering and Remote Sensing, 45(6): 727-734.

[9] Novak, K. 1992. Rectification of digital imagery, Photogrammetric Engineering and Remote Sensing, 58(3): 339-344.

[10] Skarlatos, D., 1999. Orthophotograph production in urban Areas, Photogrammetric Record, 16(94): 643-650.

[11] Catmull, E., 1974. A Subdivision Algorithm for Computer Display of Curved Surfaces, Ph.D. dissertation, Department of Computer Science, University of Utah, Salt lake city, Utah.

[12] Amhar, F., J. Josef, and C. Ries, 1998. The generation of true orthophotos using a 3D building model in conjunction with a conventional DTM, International Archives of Photogrammetry and Remote Sensing, 32(Part 4): 16-22.

[13] Rau, J., N. Chen, and L. Chen, 2000. Hidden compensation and shadow enhancement for true orthophoto Generation, Proceedings of Asian Conference on Remote Sensing 2000, 4-8 December, Taipei, unpaginated CD-ROM.

[14] Rau, J., N. Chen, and L. Chen, 2002. True orthophoto generation of built-up areas using multi-view images, Photogrammetric Engineering and Remote Sensing, 68(6): 581-588.

[15] Sheng, Y., P. Gong, and G. Biging, 2003. True orthoimage production for forested areas from large-scale aerial photographs, Photogrammetric Engineering and Remote Sensing, 69(3): 259-266.

[16] Zhou, G., 2005. Urban Large-scale Orthoimages Standard for National Orthophoto Program, XXVth IEEE International Geoscience and Remote Sensing Symposium Proceedings, 25-29 July, Seoul, Korea, unpaginated CD-ROM. 